\title{
Interoperable Geographic Information Services to Support Crisis Management
}

\author{
Artur Rocha $^{1}$, Bojan Cestnik ${ }^{2,3}$, and Marco A. Oliveira ${ }^{1}$ \\ 1 INESC Porto, R. Dr. Roberto Frias 378, 4200-465 Porto \\ \{artur.rocha, mao\}@inescporto.pt \\ 2 Temida d.o.o., SI-1000 Ljubljana, Dunajska cesta 51, \\ bojan.cestnik@temida.si \\ 3 Jozef Stefan Institute, SI-1000 Ljubljana, jamova 39
}

\begin{abstract}
In this article we focus on interoperable geographic information (GI) services from the crisis management perspective. Based on Open Geospatial Consortium [10] standards and initiatives, we present the building blocks of the interoperable solution for supporting crisis management that is proposed as a result of the EU sponsored project, MEDSI [1]. In particular, we focus on the application and operationalization of several OGC standards, some adopted and some still under discussion, such as WMS, WFS, WMC, SLD and SMS, as well as their integration and cooperation within a single software framework.
\end{abstract}

\section{Introduction}

Nowadays, we face a considerable increase in the complexity of the living environment of the western world. This trend is particularly evident in the domain of critical infrastructures. One of its negative consequences is manifested by the fact that the society has become more vulnerable. For that reason, advancing the field of crisis management for protecting critical infrastructures has been recognized as one of the top priorities in European countries 20].

One of the main concerns when responding to a crisis is how to facilitate the integration of information from various sources through different media in a meaningful way. Here, the crucial task is how to obtain timely and accurate geospatial information to quickly visualize and understand the context of emergency situations. To effectively handle this task, Open Geospatial Consortium [10] (OGC) established standards for Geographic Information (GI) processing. Among others, they promote standards like Web Map Server [9] (WMS) to retrieve geographic information in image format and Web Feature Server [12] (WFS) to retrieve GI in vector format through the use of Geography Markup Language [5] (GML). Furthermore, OGC also proposes standards that cover the display of symbols in WMS, like Styled Layer Descriptor [4, which can be found by means of a Style Management Service [6] and standards that cover the storage of a description of the requests of one or more maps from one or more map servers in a portable, platform-independent format - Web Map Context Documents (WMC) [13. Although the adoption of the OGC standards by 
GIS software providers has been a slow process, these standards are widely accepted by the GI community and represent a firm foundation for constructing distributed GI-based software systems.

The incorporation of state of the art information technology advances in the field of supporting and enhancing decision-making capabilities in crisis management represents one of the key aspects of EU sponsored project MEDSI. Since good technology is always built on the foundation of good technology, we made a commitment to apply and operationalize the standards and initiatives of OGC to propose a modern interoperable infrastructure for supporting crisis management within MEDSI. By enforcing the OGC standards, MEDSI intends to exploit in particular the interoperability issues raised by the multiplicity of formats and heterogeneous ways to access various sorts of data.

To promote the protection of critical infrastructures as one of its key areas, OGC has established a specific line of action for the Critical Infrastructure Protection Initiative 11. MEDSI has adopted this experience by incorporating many of the premises and concepts of this initiative. By building upon their work, MEDSI expects to be able to contribute for accelerating the launch of the assets of the European management decision support for critical infrastructures.

The resulting MEDSI framework was tested on realistic user scenarios, like, for example, fire and explosion of hazardous materials in an industrial area, and river flooding. The scenarios allow MEDSI partners to validate not only the suitability of the proposed architecture but also the application of instantiated MEDSI system in a real user environment. Potential users of the proposed software framework are private and governmental organizations, including city management, regional management, central institutions and agencies, international crisis management organizations, public safety and security forces, and intelligence services.

In the paper we first present the proposed MEDSI framework for supporting crisis management decisions. Then, we give an overview of technological platform. Next, the functionality of the framework is demonstrated in supporting collaborative crisis management and symbology. We conclude by calling attention to the most important points presented in this paper.

\section{Crisis Management Support}

Given the broad scope of the concept "Critical Infrastructure" and the wide variety of potential users for MEDSI system (local, regional, national or transnational level), it was obvious from the feasibility and scalability point of view that a distributed network of self-contained cells would have to be put in place. Moreover, these cells, which can vary in size and geographic distribution, need to be self-sufficient in the protection of the infrastructure they are aimed to protect, while they also should be able to cooperate in case of broader emergency situations [Figure 1].

Also, the central role of GI together with the ability to swiftly exchange the most updated representations of geographical data (maps) among crisis manage- 
ment actors was a premise in the inception of MEDSI. This assumption remains true not only for exchanges between MEDSI cells, but also inside the scope of a single cell, instantiated in a crisis management center.

Originating from these premises and being aware of the interoperability issues arising when trying to use together several heterogeneous GIS software and data sources, we have decided to enforce the use of OGC Web Services (OWS) for all sorts of GI access and exchange in MEDSI.

Users of MEDSI system have the capability of finding the appropriate GI sources by means of a catalogue service where the Web Services providing the relevant geodata have been previously registered.

It should be stressed that the services providing GI may be available not only inside the crisis center, where typically resides information like local aerial photos, streets, buildings, available resources, etc., but also from external providers like up to date satellite photos, sensor/weather data or even the most updated "map" of an endangered facility, made available upon request [figure 1].

Although this paper will not focus on the specific use cases implemented in MEDSI, it's worth mentioning that it consists of a rich client application capable of providing support to domain-specific tasks such as risk analysis, crisis plans, standard operation procedures, etc. In other words, MEDSI handles a set of specific domain objects, some of them comprising associated geometries, and it tries to do so maintaining the lowest possible coupling with the datasource level. For the GI realm, this means that MEDSI domain objects can be accessed through a WFS interface. As such, it is possible that another web service

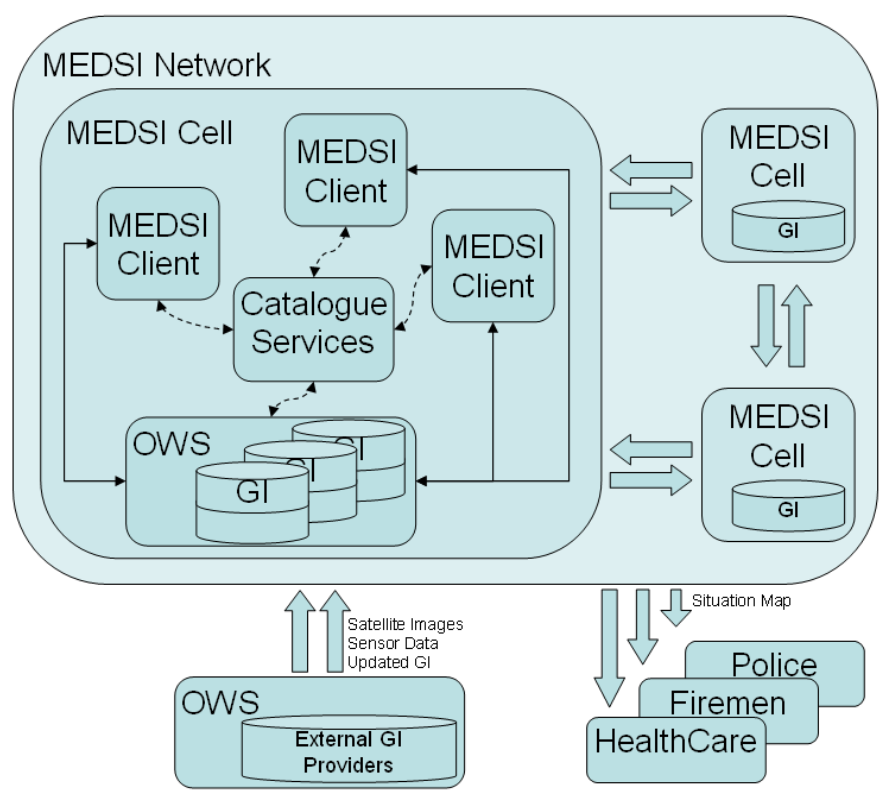

Fig. 1. Organizational view of MEDSI 
makes a "request" to the WFS for its schema in order to be able to interoperate with it.

As outputs from MEDSI system, the most recent "situation maps" are produced to generate reports and updates to the units operating in the crisis scene, as well as for leading updated information to the several actor responsible for taking decisions in a crisis situation.

Moreover, it's worth mentioning that due to the ability of exchanging "Context Documents" amongst crisis management actors, these can interact in a much more efficient way as they are able to see exactly the same "map representation" (as if using paper based maps), but with the possibility of continuing to browse afterwards like in any on-line map.

\section{Technology Overview}

MEDSI comprises several modules supporting crisis management specific tasks ranging from Analysis and Planning to Simulation, Decision Support, Resources Management and also some horizontal types of functionalities like messaging and reporting.

These modules which implement the main specific functionalities will not be detailed under the scope of this paper, however they have the particularity of being connected to the GI infrastructure in one way or another. First because objects addressed in the crisis management domain correspond directly to a geographical feature. And second, because although sometimes no direct mapping is feasible, the geographical representation of involved areas provides a meaningful context to understand and respond to a crisis situation or help revealing a potential threat.

A special attention has also been paid to symbology, given its high expressiveness which helps the user to quickly absorb a significant level of information, provided that the used symbols are commonly understandable.

In this section we describe the technological platform being built for MEDSI project focusing mainly on the GI infrastructure.

As previously said, MEDSI has chosen to use in its prototype WMS as a portrayal service for displaying maps in image format and WFS for data services returning GML, which is then rendered in vector format. Because aerial photographs provide a high level of understandability even for users not familiarized with cartography, a Web coverage Service (WCS) [7] was also used in MEDSI prototype realization.

Although other services such as Web Terrain Server (WTS) [2] could also have been used for enhanced terrain visualization, the aforementioned ones have been deemed adequate for establishing a proof of concept.

As the first step in the prototype implementation, the consortium has configured several geographical data sources, in different technologies capable to output GI under the form of WMS and WFS. MEDSI has successfully configured and tested interoperable access to several platforms providing GI by means of web services. From then the consortium has been seamlessly using 
both open source geodata sources such as Deegree [14, UMN map server [17] and Geoserver [18], but also popular proprietary solutions such as Geomedia Web Server [16] and ESRI ArcIMS [15] through their respective WMS and WFS connectors.

A catalogue service has also been deployed [8] and enhanced with the capability of classify these GI services according to a proposed ontology to facilitate finding of the appropriate data sources.

On MEDSI client side, we used an Open Source GI viewer 19] able to view WMS which we extended to support WFS accesses as well as other functionalities required for proper integration with other implemented modules.

A catalogue browser is also used in the client for finding the needed data sources. Some editing and annotation functionalities, along with some basic spatial analysis have also been used to provide added value to MEDSI client [figure2].

Although the resulting prototype has proved itself as a reliable, highly customizable and interoperable framework for accessing distributed geographical information, other functionalities have been identified as a prerequisite for supporting a collaborative approach to decision support in crisis management.

One of such functionalities is the ability to store and further reproduce the status of a GI view, i.e. the set of map requests that originated that same view. Saving and reproducing map context information is essential to support any underlying workflow mechanism and also to create personal views for each user profile or for each type of crisis.

Furthermore, a gazetteer service [3] can be used to find a geographic feature by its name (e.g. a street) returning a new geographical extent to update the present context.

Other important factor to support collaboration is the ability of crisis management actors within a cell to be able of visualizing geographic information

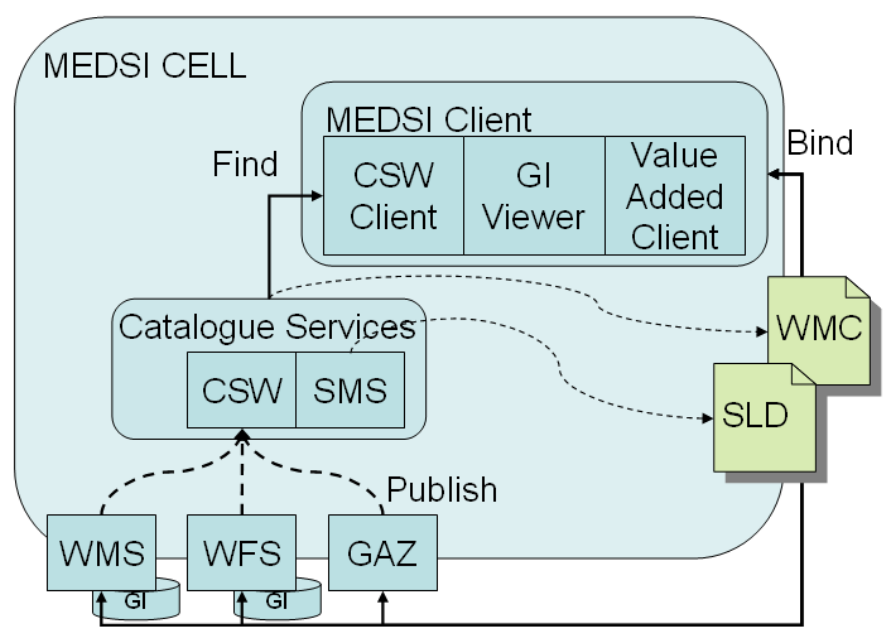

Fig. 2. OGC Web Services at use in MEDSI 
from other cells, e.g. points of interest, according to their own used symbology, leading to the requirement of changing symbology in runtime.

\section{Supporting Collaboration}

A successful collaborative approach starts with the definition of each actor's roles and needs. In this sense, MEDSI allows the definition of a set of user profiles associated with the actors' application needs and preferences. Depending on the type of crisis, the user may also have a pre-defined set of default information needs.

The collaborative nature of MEDSI framework has turned the requirements concerning workflow mechanisms and broadcast of situation maps a priority.

From the GI infrastructure point of view, this builds up to the need of loading, saving and exchanging map contexts, containing the necessary information used to recreate the exchanged "map view".

Despite its limitation of binding only with OGC Web Map Service interface, OGC Web Map Context Documents (WMC), or simply contexts [13] were used as a starting point to fulfill these requirements.

A context is some sort of "memento" for maps, comprising the description in a portable, platform-independent format of the grouping of one or more map requests from one or more map services for storage in a repository or for transmission between clients [13].

As such, the consortium has implemented a specific framework component for loading and saving WMC documents (XML), as well as a repository for storing and retrieving them which also provides an ad-hoc workflow mechanism allowing the exchange of contexts between users and/or groups of users.

Figure 3 depicts a situation map being sent from the Head of a Crisis Management Center (HC) to the Analysts Team (AT) for further task execution. The analysts will then receive a notification that a new context document awaits them and upon opening it, their "map viewer" will be directed to the area specified by the $\mathrm{HC}$, i.e. the client will perform the same map requests necessary to reproduce the same situation map.

In another example of usage inside a specific MEDSI Cell, a context document resulting from pre-planning tasks can be bound to a crisis ontology, so that when a specific crisis situation occurs, the most suitable "map context" (servers, layers, extents, etc) will be automatically loaded.

Context documents are also used to support the broadcast of live maps, either between cells or to external actors, such as Firemen. This is important when dealing with information that is constantly evolving during the crisis, enabling the receivers to access the most up to date information or simply a more relevant view over the same geodata.

Either supported by a traditional workflow engine or simply by means of adhoc workflow support, WMC documents have an important role in enabling collaborative distributed decision support for GI-based applications such as MEDSI.

Although the current software infrastructure serves as proof of concept for collaboration by enabling the mechanisms to store and find context documents, 


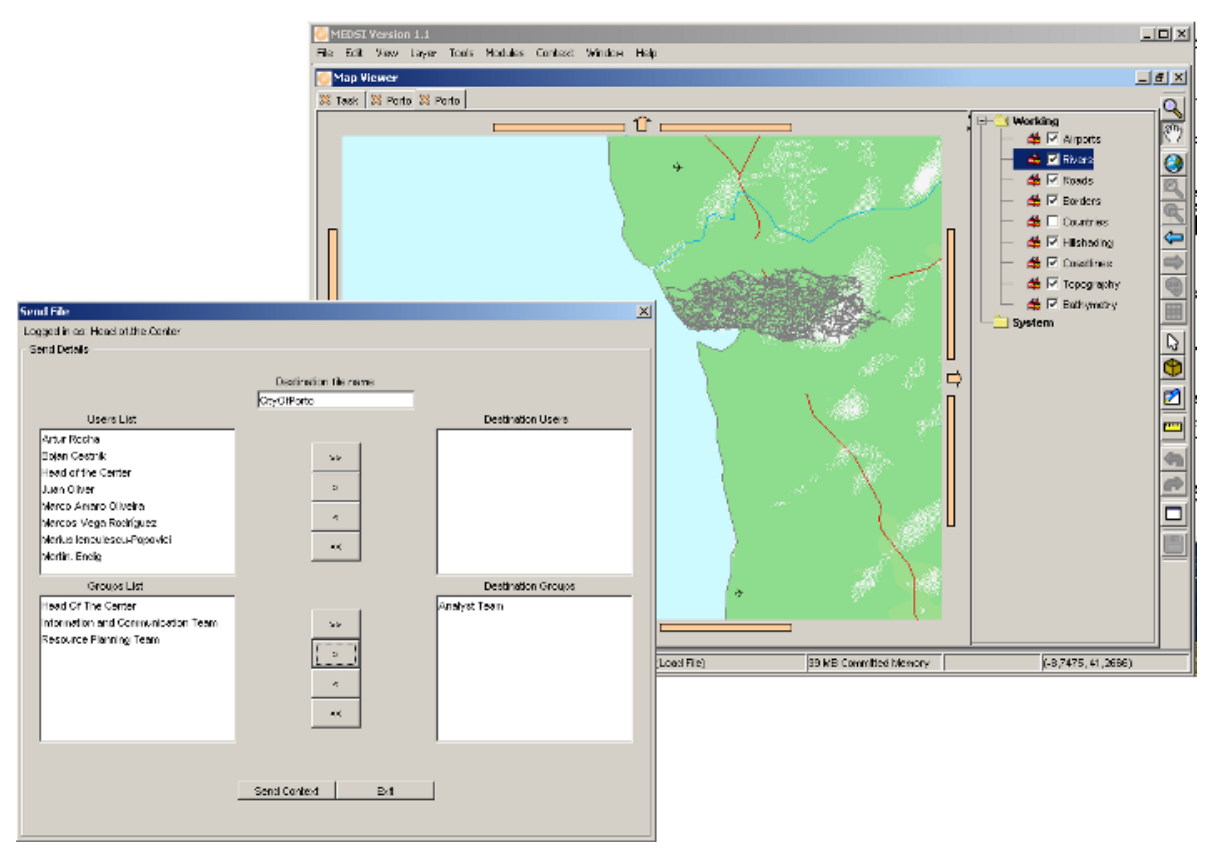

Fig. 3. Web Map Context Document - Sending

broader usage could probably benefit from a Web Catalogue Service, eventually leading to a spatial extension to Universal Description, Discovery and Integration (UDDI).

Since Web Map Context Documents have the limitation of only binding with WMS interfaces, empowering its usage will require further extensibility in order to bind with other OGC interface specifications such as WFS.

\section{$5 \quad$ Symbology}

A collaborative approach to crisis management requires establishing a common "language" for team communication. Symbology takes an essential role for quick visual identification of the most important spots within the crisis geographical extent. Within MEDSI project we have established a framework for the generation of symbols from a structured definition containing the symbol description.

Styled Layer Descriptor 4 is a language that can be used to customize the output of WMS and WFS on the client side, as it defines styles for presenting different map layers. A symbol for denoting a specific phenomenon on the map is first dynamically constructed from an icon selected from the symbology repository and augmented with dynamic information from the data base. Then, the necessary SLD file is built and placed in a location accessible from the web. 
Since the SLD location (URL) is a paramenter in a WMS request, new WMS requests using the stylesheet (SLD) at that location will display the updated symbols on the map. In such a way, symbols can be displayed with dynamical attributes of the portrayed entity.

On the other hand, since for each WMS entry in the catalogue service MEDSI defines a collection of possible SLDs, it is possible to have a different set of symbols for each "user community", which facilitates communication while they don't agree on a common set.

Style Management Service (SMS) manages objects that represent styles and symbols and provides the means to discover, query, insert, update and delete these objects. Styles provide the mapping from feature types and feature properties and constraints to parameterized symbols used in drawing maps. Typically, SMS is realized by means of a catalogue service and provides a way of finding an appropriate SLD for displaying symbols on a WMS. By the same token, both technologies are used also within MEDSI framework.

\section{Lessons Learned}

MEDSI has decided to follow the path of OGC Web Services for its own GI infrastructure, while aiming to support a distributed and collaborative approach to Crisis Management. This has proved a strong asset concerning the aspect of solving interoperability issues that necessarily arise when using distributed heterogeneous systems and data sources.

By enforcing the use of standards like WMS and WFS to help solving issues resulting from the definition of a distributed architecture, MEDSI has aligned itself with the European and International tendencies on building common Spatial Data Infrastructures, which can help solving relevant problems such as the ones involved in the protection of critical infrastructures.

The definition of a solid application layer for a specific domain, over a set of distributed geodata sources in the form of web services has brought MEDSI before the need of complementing the standard interfaces for accessing data with many other abstractions and mechanisms to provide the necessary functionalities while keeping independence from the information sources.

An example of these abstractions was a kind of context information that could be used to save, exchange and restore the status of a composed GI view. The Web Map Context documents (a position paper by the time we started) was identified as the vehicle to store and transport this information across the network, thus enabling collaboration.

Symbology can be seen as lingua franca for interpreting emergency maps, however different communities may use different symbols to represent the same concepts. Dynamic binding of Styled Layered Descriptions (SLD) to map services (WMS) has helped overcome this issue.

On the other hand, the low-coupling between maps services and symbols and the fact that they can be loaded in run-time has helped MEDSI taking the dynamic aspects of symbology a step further into augmenting symbols with database information for better and faster map interpretation. 


\section{Acknowledgement}

The work reported in this article is a result of the research project "MEDSI IST-2002-506991" funded by the Commission of European Communities. We are grateful to all the MEDSI Consortium members for their contributions to the project.

\section{References}

1. Bojan Cestnik, Artur Rocha, and Martin Endig. Emergency response through collaborative crisis management. In Proceedings of Eastern European eGov days 2005, Budapest, Hungary, March 17-18 2005.

2. Open Geospatial Consortium. Web terrain server, version 0.3.2. Available in World Wide Web, August, 24 2001. http://portal.opengeospatial.org/files/ ?artifact_id=1072.

3. Open Geospatial Consortium. Gazetteer service profile for a wfs, version 0.0.9. Available in World Wide Web, September, 03 2002. https://portal. opengeospatial.org/files/?artifact_id=7175.

4. Open Geospatial Consortium. Styled layer descriptor implementation specification, version 1.00. Available in World Wide Web, August, 19 2002. https://portal. opengeospatial.org/files/?artifact_id=1188.

5. Open Geospatial Consortium. Geography markup language, version 3.0. Available in World Wide Web, January, 29 2003. https://portal.opengeospatial.org/ files/?artifact_id=7174

6. Open Geospatial Consortium. Style management services for emergency mapping symbology, version 1.00. Available in World Wide Web, January, 202003. http://portal .opengis.org/files/?artifact_id=7470

7. Open Geospatial Consortium. Web coverage service, version 1.0. Available in World Wide Web, October, 16 2003. https://portal.opengeospatial.org/ files/?artifact_id=3837

8. Open Geospatial Consortium. Catalog service specification, version 2.0. Available in World Wide Web, August, 02 2004. http://portal.opengeospatial.org/ files/?artifact_id=5929\&version=1.

9. Open Geospatial Consortium. Web map service specification, version 1.3. Available in World Wide Web, August, 02 2004. http://portal.opengeospatial.org/ files/?artifact_id=5316.

10. Open Geospatial Consortium. Available in World Wide Web, 2005. http://www . opengeospatial.org.

11. Open Geospatial Consortium. Critical infrastructure protection initiative. Available in World Wide Web, 2005. http://www.opengeospatial.org/functional/ ?page=cipi

12. Open Geospatial Consortium. Web feature service specification, version 1.1. Available in World Wide Web, May, 03 2005. https://portal.opengeospatial.org/ files/?artifact_id=8339.

13. Open Geospatial Consortium. Web map context documents, version 1.1. Available in World Wide Web, May, 03 2005. https://portal.opengeospatial.org/files/ ?artifact_id=8618.

14. Deegree. Available in World Wide Web, 2005. http://deegree.sourceforge.net/ 
15. ESRI. Gis standards and it interoperability. Available in World Wiede Web, 2005. http://www.esri.com/software/standards/

16. Intergraph. Geomedia. Available in World Wide Web, 2005. http://www . intergraph.com/geomedia/

17. University of Minnesota MapServer. Available in World Wide Web, 2005. http://mapserver.gis.umn.edu/

18. The GeoServer Project. Available in World Wide Web, 2005. http://geoserver.sourceforge.net/

19. The JUMP Project. Available in World Wide Web, 2005. http://www . jump-project.org/

20. J. Solana. A european route to security. The International Herald Tribune, December 12 2003. Also published in other international newspapers. 